\title{
Improvement of the Wavelength Switching Behavior of Semiconductor Tunable Lasers Through Optical Feedback From a Periodic Reference Filter Based on a Novel Prism-Based Implementation of a Fox-Smith Resonator
}

\author{
Bart Moeyersoon, Geert Morthier, Senior Member, IEEE, Ronny Bockstaele, Member, IEEE, and \\ Roel Baets, Senior Member, IEEE
}

\begin{abstract}
We show that the wavelength switching behavior of semiconductor tunable lasers can be improved through optical feedback from a stable reference filter. The filter is based on a novel prism-based implementation of a Fox-Smith resonator and has a response consisting of periodically spaced peaks, both in reflection and transmission. The improvement of the wavelength switching behavior stems from the suppression of the thermally induced wavelength drift associated with the switching of the tuning currents.
\end{abstract}

Index Terms-Optical communication, optical switching, tunable lasers.

\section{INTRODUCTION}

$\mathbf{I}$ $\mathrm{N}$ ORDER to avoid the electronic bottleneck of current network routers and to have a more flexible and efficient usage of the very high bandwidth capacities of the (dense) wavelength-division-multiplexing technology, it is expected that future network technologies will move toward optical packet/burst switching [1]. This will help create the so-called "all-optical" network. Obviously, rapidly (in the order of $10 \mathrm{~ns}$ ) tunable transmitters will be one of the key components for such a technology. Some semiconductor tunable lasers allow such rapid electronic tuning [2], but then one encounters problems on a larger time scale due to the thermally induced wavelength drift [3]-[4].

From theoretical considerations and numerical simulations, it is known that this drift shows an exponential decay that is dominated by discrete time constants [3]. The wavelength switching behavior can, thus, be improved by somehow suppressing these exponential terms in the wavelength drift. A possible method to do this is to (pre)compensate the tuning currents for each of the three time constants by simply adding resistance-capacitance pairs to the current drive network of the tuning sections [4]. Unfortunately, due to the quadratic dependence of the heating on current $\left(\sim \mathrm{RI}^{2}\right)$, compensation for different current step values is difficult to obtain using only linear elements. In this letter,

Manuscript received April 21, 2005; revised June 9, 2005. This work was supported by the DWTC through the IAP5/18 Project, and by the European Union in the context of the IST Project STOLAS.

The authors are with the Department of Information Technology, Ghent University-IMEC, Gent B-9000, Belgium (e-mail: bmoeyers@intec.ugent.be).

Digital Object Identifier 10.1109/LPT.2005.856384 we present a method, based on optical feedback from a stable reference filter, that can be used to suppress the thermally induced wavelength drift independent of the size of the current step; i.e., the compensation is independent from which channel the wavelength is being switched to and also independent from the switching history.

\section{PRINCIPLE}

The solution we propose is to provide a stable optical reference the laser wavelength can be stabilized to by optically coupling the laser and the reference. There are a few obvious requirements the optical reference must fulfil. The first one is that it should provide a stable reference for each of the wavelengths the tunable laser is supposed to work at. For a telecommunications network, these wavelengths (or optical frequencies) lie on the ITU grid, i.e., a set of frequencies anchored on $193.1 \mathrm{THz}$ and periodically spaced by $100 \mathrm{GHz}$ or for future networks even 50 or $25 \mathrm{GHz}$. Another requirement is that the reference frequencies are independent of the thermal condition of the laser. This can easily be obtained by physically separating the frequency reference from the laser. This reasoning gave rise to an optical feedback scheme where the optical frequency reference is provided by a common Fabry-Pérot etalon that is optically coupled to the laser by two 3-dB power splitters [5]. In [5], it was numerically shown that a wavelength drift of $15 \mathrm{GHz}$ can be suppressed in this way. The laser used in the simulations was a three-section distributed Bragg reflector (DBR) laser with an antireflection (AR)-coated facet $(R=0.01)$ so that the feedback level was high enough to avoid instabilities caused by the optical feedback. A sufficiently strong feedback level (typically more than $-10 \mathrm{~dB}$ ) together with a short external cavity (typically less than $10 \mathrm{~cm}$ ) is required to obtain stable external cavity operation [6].

Practical implementation of the scheme from [5] without destabilizing the laser is hindered by the presence of the two power splitters, making it difficult to obtain a high feedback level and a short external cavity. These two splitters are required because existing comb filters like Fabry-Pérot etalons only have a comb-like response in transmission, while the response in reflection has a complementary form with dips in stead of peaks at the ITU-frequencies. There are filter types (for instance 


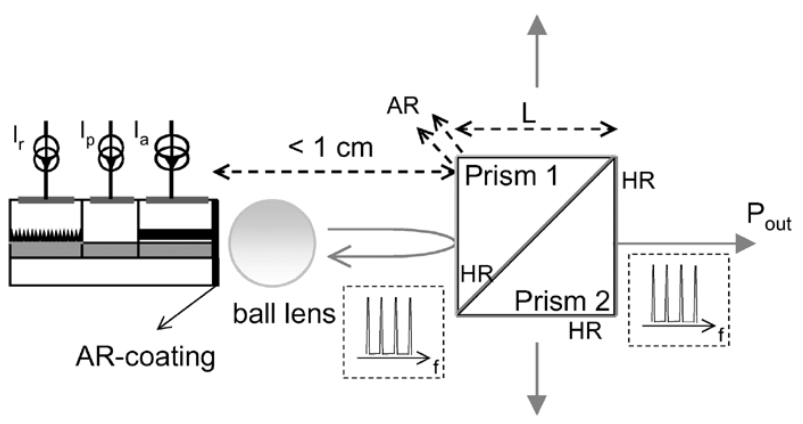

Fig. 1. Schematic representation of the coupling of a semiconductor (tunable) laser with the filter through a ball lens (diameter $=2 \mathrm{~mm}$, focal distance $=$ $0.11 \mathrm{~mm}$ ).

based on fiber Bragg gratings) that have a comb-like response in reflection, but since these filters have the complementary response with dips in transmission, one still needs one extra power splitter to obtain a reasonable output power from the laser. Furthermore, fiber Bragg grating based filters are usually quite long (a few centimeters or more).

\section{DESCRIPTION OF FILTER}

For the reasons mentioned above, we opted to develop a filter with peaks at the same (ITU) frequencies both in reflection and transmission and that is as short as possible. This filter is based on a novel prism-based implementation of a Fox-Smith resonator [7]. The filter consists of two right-angle prisms with refractive index $n$ and base length $L$, cemented together with ultraviolet-curable index-matching glue (a schematic representation of the filter can be seen on the right-hand side of Fig. 1). The only function of the upper prism is that the incident light can be horizontal for the beams reflecting in the lower prism to be perpendicular to the right facets of the lower prism and this without having to worry about total internal reflection on the air-glass interface (when the upper prism would not be present). For this reason, the front facet of the upper prism should be AR coated. After propagation through the upper prism, the beams will undergo multiple reflections in the bottom prism and the different reflections will interfere constructively or destructively depending on the frequency of the light, just like in a normal Fabry-Pérot etalon, except that in our structure there are three mirrors instead of two, namely the three facets of the lower prism. As in a classical Fabry-Pérot etalon, the mirrors on these three facets should be highly reflective in order to have a high finesse filter. To avoid that the first reflection of the incident light on the diagonal facet of the lower prism would reflect at the top facet of the upper prism, this facet should be AR-coated. The free spectral range (FSR) of the filter is determined by the refractive index $n$ and the base length $L$ of the prisms and is given by FSR $=c / 2 n L$, with $c$ the speed of light. It should be noted that the filter basically acts as a four-port device. The transmission to the (unused) output port at the top facet has periodic dips, while the transmission to the (unused) bottom facet port is similar in behavior to the transmission to the right facet. These output ports can be used for monitoring purposes so that an extra wavelength locker filter would not be required.

Fig. 2 shows the experimentally determined reflection and transmission response of the novel filter type. The prisms that we

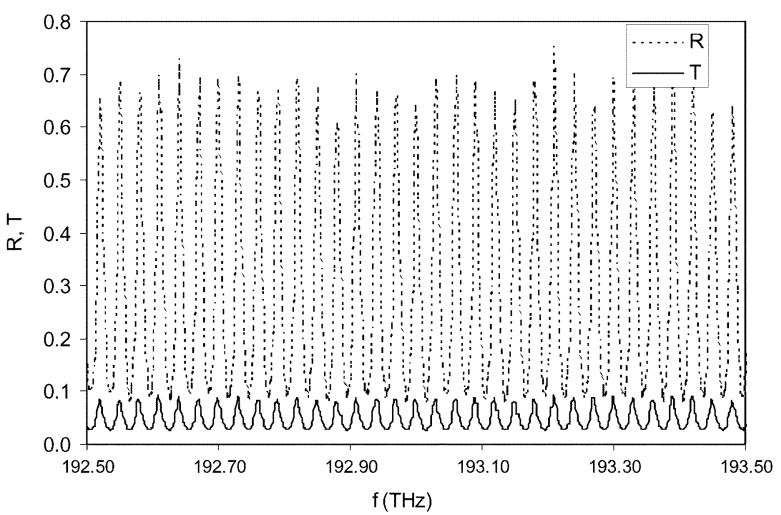

Fig. 2. Experimentally determined reflection (broken line) and transmission (full line) response of the novel filter type.

used were standard available high-precision right-angle prisms made of BK7 material and with a base length of $3.2 \mathrm{~mm}$. This gives rise to an FSR of around $32.5 \mathrm{GHz}$. The smallest available standard size for these high-precision right-angle prisms is $3.2 \mathrm{~mm}$. In a network environment, a prism base length that corresponds with an FSR of $100 \mathrm{GHz}$ (or $50 \mathrm{GHz}$ ) would be more desirable. In that case, the base length would be around 1 and $2 \mathrm{~mm}$, respectively, and one would require custom-made prisms. The reflectivity of the different coated facets was $R_{\mathrm{AR}}=0.0127$ for the two right facets of the top prism, $R_{\mathrm{HR}}=0.98$ for the two right facets of the bottom prism, and $R_{S}=0.7$ for the diagonal facet of the bottom prism). From Fig. 2, it can be seen that both the reflection and transmission response have a comb-like behavior, i.e., they consist of a periodically spaced set of frequency peaks. The transmission peaks are so small, because the different reflection coefficients were chosen to have a sufficiently high reflection (on the filter peaks) back to the laser in order to be certain that we would obtain stable external cavity operation. Other values for the reflection and transmission (e.g., both 50\% on the peaks) can be obtained by properly choosing the reflection coefficients of the coatings on the different facets.

\section{EXPERIMENTAL RESULTS}

Fig. 1 shows a schematic representation of the coupling of the laser with the filter through a ball lens. The laser is a three-section DBR tunable laser with an AR-coated facet and an output power of $5 \mathrm{~mW}$ for an active current $I_{a}$ of $100 \mathrm{~mA}$. The ball lens has a diameter of $2 \mathrm{~mm}$ and a focal distance of $0.1 \mathrm{~mm}$. Both the ball lens and the prisms of the filter are low-cost standard components that are readily available. By developing this new filter type, we were able to keep the external cavity length below $10 \mathrm{~cm}$. Even with a short external cavity, it is still required to ensure a sufficiently high feedback level. Therefore, good coupling of the laser with the filter is very important. Taking into account that the minimum coupling loss for the light from the laser to the filter and back into the laser will be $3 \mathrm{~dB}$, we estimate that the feedback level ranges between -5 and $-7 \mathrm{~dB}$ (near the filter peaks).

Fig. 3 shows a part of the experimentally obtained tuning table of the external cavity laser shown in Fig. 1. The lasing frequency (squares) and the sidemode suppression ratio (SMSRtriangles) are shown versus the current $I_{r}$ through the reflector section for $I_{a}=100 \mathrm{~mA}$ and a phase current $I_{p}=2 \mathrm{~mA}$. It can be seen that the frequency plateaus are horizontal contrary 


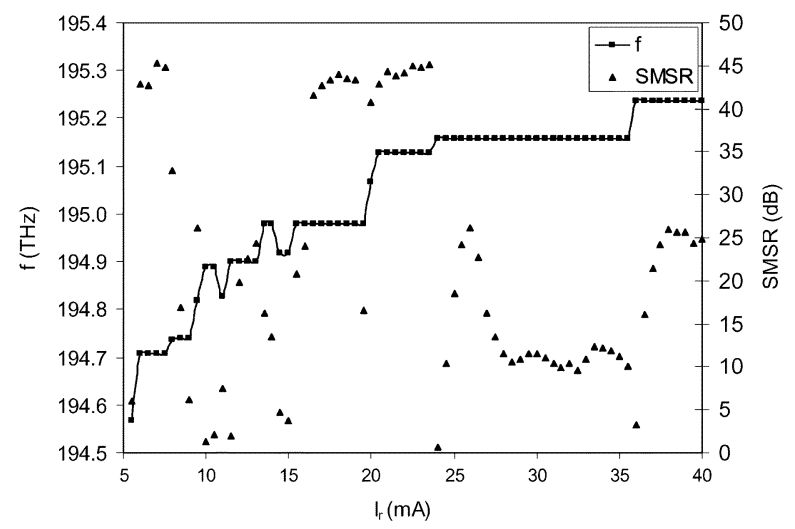

Fig. 3. Excerpt from the experimentally obtained tuning table. The lasing frequency (squares) and the SMSR (triangles) are shown versus $I_{r}$ for $I_{a}=100 \mathrm{~mA}$ and $I_{p}=2 \mathrm{~mA}$.

to the (slightly) tilted plateaus of a free-running DBR laser. This is because the frequency of each plateau is now determined by a fixed peak of the filter, while for a free-running DBR laser, it is determined by the (quasi-)continuously shifting Bragg peak. The difference between the plateaus is (a multiple of) the FSR of the filter, whereas for a free-running DBR laser this is the cavity-mode spacing. The intermediate frequency jumps (e.g., around $I_{r}=11 \mathrm{~mA}$ ) and the fact that the SMSR is not above $40 \mathrm{~dB}$ for every frequency plateau can both be attributed to the small FSR of the filter. This FSR is only $32.5 \mathrm{GHz}$ and since the $3-\mathrm{dB}$ width of the Bragg peak is typically $100 \mathrm{GHz}$ or more, there are always at least three filter peaks lying within the Bragg peak. The round-trip gain is, thus, nearly the same for these filter peaks, so that two or more laser modes can coexist at the same time (yielding a low SMSR in some tuning current regions, e.g., between 25 and $35 \mathrm{~mA}$ ). The coexistence of several laser modes also means that minor (parasitic) deviations in, e.g., the external cavity length can cause uneven frequency jumps when the reflector current is tuned. This can be greatly improved using a filter with an FSR that is better adapted to the width of the Bragg peak (e.g., an FSR 50 or $100 \mathrm{GHz}$ ) so that fewer filter peaks lie within the Bragg peak at the same time. However, since our goal is to prove that the wavelength switching behavior can be improved using this principle, we only need two stable operating points with high SMSR to switch between. We also want to stress that the tuning table was recorded without any control loop applied to the laser as is normally the case. This is also a reason why the SMSR is not above $40 \mathrm{~dB}$ for all frequency plateaus. By properly adjusting the tuning currents, it is possible to obtain an SMSR above $40 \mathrm{~dB}$ for every filter peak within the tuning range of the laser. An extra advantage is that it is easier to build a tuning table for a tunable laser that is stabilized to an (external) frequency reference, since one no longer has to search for the desired frequencies in the frequency versus tuning currents plane. Furthermore, aging of the tuning sections or tuning table inaccuracies will have a reduced influence.

The maximum output power after the filter for each frequency plateau lies around $1.4 \mathrm{~mW}$. This value critically depends on the beam quality within the Fox-Smith resonator and, therefore, on the lens quality. Replacement of the simple ball lens by a high quality lens is expected to increase the output power considerably.

Fig. 4 shows experimentally obtained traces of the (absolute value of the) deviation of the laser wavelength from its steady-

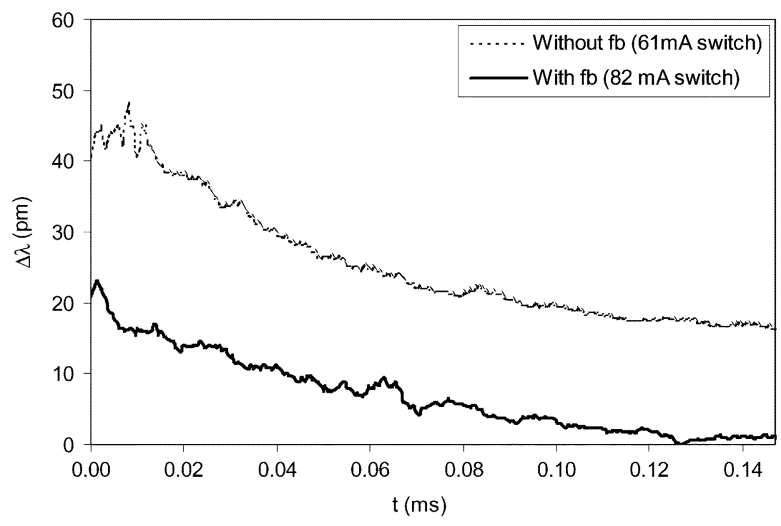

Fig. 4. Experimentally obtained traces of the deviation of the laser wavelength from its steady-state value after switching, both with and without feedback from the filter. $I_{r}$ was switched between 0 and $61 \mathrm{~mA}$ (broken line) for the case without feedback and between 0 and $82 \mathrm{~mA}$ (thick line) for the case with feedback.

state value after switching, both with and without feedback from the filter. The reflector current was switched at $t=0 \mathrm{~ms}$ between 0 and $61 \mathrm{~mA}$ (broken line) for the case without feedback and between 0 and $82 \mathrm{~mA}$ (thick line) for the case with feedback. The active current was $100 \mathrm{~mA}$ in both cases and the phase current was adjusted for maximum SMSR each time. It can be clearly seen that the drift is greatly reduced by the feedback, even though the switching current is $30 \%$ higher compared to the situation when no feedback is present. The maximum deviation is reduced and the steady-state value is reached much faster. Without feedback the steady-state is not reached before $2 \mathrm{~ms}$ after the switch. The fact that no complete suppression of the thermal drift is obtained is again due to the low FSR of the filter.

\section{CONCLUSION}

We have shown that the wavelength switching behavior of a semiconductor tunable laser can be improved by stabilizing the laser at (the peaks of) a periodic reference filter. A novel filter was devised and fabricated for this purpose. Further improvement can be obtained by better adapting the FSR of the filter to the width of the Bragg peak of the laser. The use of the filter will also make it easier to generate tuning tables and will reduce the influence of ageing or of tuning table inaccuracies.

\section{REFERENCES}

[1] P. Green, "Progress in optical networking," IEEE Commun. Mag., vol. 39, no. 1, pp. 54-61, Jan. 2001.

[2] O. A. Lavrova, L. Rau, and D. J. Blumenthal, "10-Gb/s agile wavelength conversion with nanosecond tuning times using a multisection widely tunable laser," J. Lightw. Technol., vol. 20, no. 4, pp. 712-717, Apr. 2002.

[3] M. Teshima, "Dynamic wavelength tuning characteristics of the $1.5-\mu \mathrm{m}$ three-section DBR lasers: Analysis and experiment," IEEE J. Quantum Electron., vol. 31, no. 8, pp. 1389-1400, Aug. 1995.

[4] E. G. Vicente, D. Vera, and B. Glance, "Switching-time limitations in tunable multisection lasers," IEEE Photon. Technol. Lett., vol. 2, no. 11, pp. 781-783, Nov. 1990.

[5] B. Moeyersoon, G. Morthier, and R. Baets, "Novel optical feedback scheme for improvement of the wavelength switching behavior of tunable lasers," in Proc. 2002 IEEE 18th Int. Semiconductor Laser Conf., pp. 91-92.

[6] K. Petermann, "External optical feedback phenomena in semiconductor lasers," IEEE J. Sel. Topics Quantum Electron., vol. 1, no. 6, pp. 480-489, Jun. 1995.

[7] P. W. Smith, "Stabilized, single-frequency output from a long laser cavity," IEEE J. Quantum Electron., vol. QE-1, no. 8, pp. 343-348, Nov. 1965. 